\title{
Novel Time-Resolved Fluorescence Microscope System using TCSPC and Multi-frequency techniques
}

\author{
L.L. Chandler and J.R. Mattheiss \\ Fluorescence Division, Horiba Jobin Yvon, Inc,, 3880 Park Ave., Edison, NJ 08820
}

Time-resolved fluorescence microscopy is the ultimate tool to study dynamic events in cellular structures and nano-materials. Unlike the intensity based fluorescence microscopy, fluorescence lifetime is an intrinsic property of a fluorophore, and its measurement does not suffer any interference caused by changes in fluorescence intensity, such as photo-bleaching, excitation light instability, and light scattering. More importantly, time-resolved measurements can provide essential dynamic information about a fluorophore microenvironment and its interaction with other molecules. Currently, TCSPC time-domain and frequency-domain techniques are commonly used for fluorescence lifetime imaging microscopy (FLIM). HORIBA Jobin Yvon (HJY), the leader in fluorescence spectroscopy, introduces a filter-based fully automated confocal microscope system (DynaMyc ${ }^{\mathrm{TM}}$ ) to measure fluorescence lifetime and intensity in micro scales. This unique FLIM system can be configured with either TCSPC or multifrequency technique. In this paper, the merits of each time-resolved methodology will be discussed. In addition, a novel HJY multi-frequency domain lifetime system will be presented. By virtue of its ability to measure multiple frequencies simultaneously, this new multifrequency lifetime system exhibits ultra-fast data acquisition over a broad dynamic range. In contrast to single frequency measurements that provide average lifetime information, the multiple frequency measurements allow accurate resolution of individual lifetimes for complex decays in a single acquisition. This system is presented to highlight its potential to revolutionize time-resolved microscopy. 


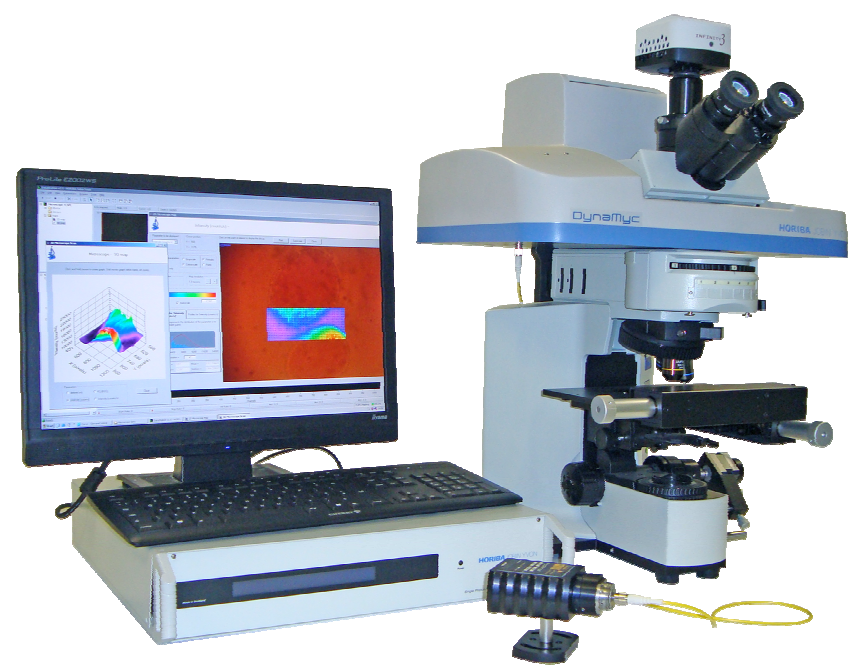

Figure 1, HJY DynaMyc ${ }^{\mathrm{TM}}$ fluorescence lifetime imaging system

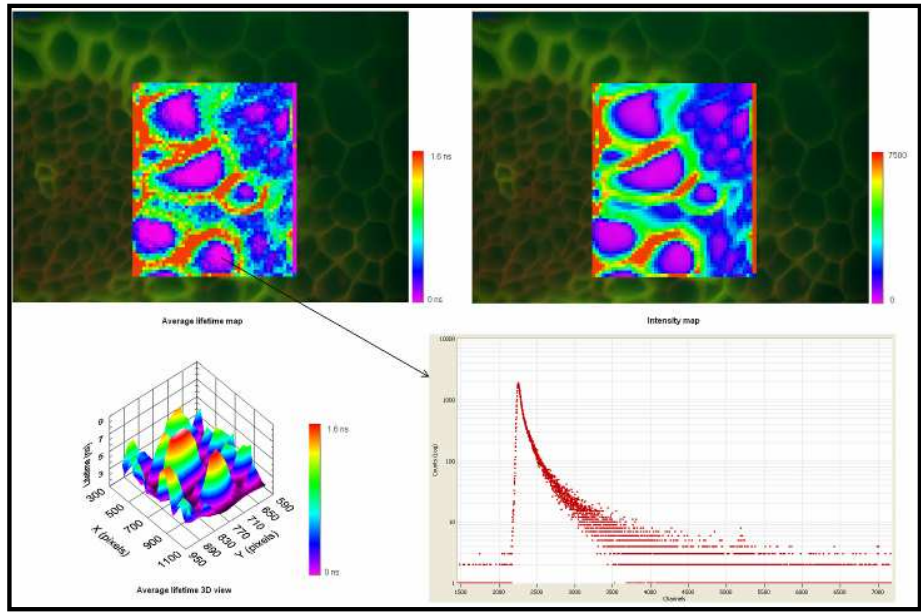

Figure 2, Lifetime and fluorescence intensity imaging on convallaria sample 